\title{
Bloqueos de Extremidad Inferior y Cirugía Ambulatoria
}

\section{Lower extremity nerve blocks for Outpatient surgery \\ https://doi.org/10.25237/carsach2020.13}

Dr. Fernando R. Altermatt C. ${ }^{1}$ Dra. Andrea Araneda V. ${ }^{1}$ Dr. Fernando Rueda B. ${ }^{1}$ Dr. Pablo Miranda H. ${ }^{1}$

${ }^{1}$ Sección de Dolor y Anestesia Regional. División de Anestesiología. Escuela de Medicina. Pontificia Universidad Católica de Chile.

Dr. Fernando R. Altermatt C.

e-mail: falterma@uc.cl

ORCID ID: https://orcid.org/0000-0002-0464-8643

Palabras claves:

Bloqueo de Nervio periférico, Extremidad Inferior, Anestesia Regional, Cirugía Ambulatoria.

Key words:

Peripheral Nerve blocks, Lower Extremity, Regional Anesthesia, Outpatient Surgery.

\section{Puntos clave}

-El uso de anestesia/analgesia regional mejorar la calidad de la recuperación y puede aumentar la eficiencia de la cirugía ambulatoria.

-Pese a lo anterior, sigue siendo una técnica sub-utilizada, en particular en cirugías de extremidad inferior.

- Las cirugías de extremidad inferior se benefician de incorporar técnicas de anestesia/analgesia regional. Sin embargo, la disponibilidad de buena calidad de evidencia avalando su uso es dispar para distintos tipos de cirugía.

- No es concebible una adecuada anestesia/ analgesia para cirugía ambulatoria sin un abordaje multimodal, en que los bloqueos de nervio periférico son un componente más.

-El riesgo potencial de complicaciones, en particular el riesgo de caídas en pacientes que han recibido anestesia/ analgesia regional periférica es un aspecto que debe ser activamente abordado y prevenido, con un enfoque de balance riesgo/ beneficio.

\section{Resumen}

El manejo del dolor perioperatorio de una manera eficiente es una condición mandatoria para poder realizar cirugía ambulatoria. Analgesia inadecuada es fuente de múltiples efectos adversos, retardo del alta, y readmisiones no programadas. El uso de técnicas regionales por tanto es una aproximación racional a la prevención y manejo de estos problemas.

Esta revisión narrativa se concentrará en revisar la evidencia disponible (si es que existe) para apoyar el uso de técnicas de anestesia/analgesia regional periférica de extremidad inferior para cirugías comúnmente realizadas en el contexto ambulatorio. 


\begin{abstract}
Efficient perioperative pain management is a mandatory condition for performing outpatient surgery. Inadequate analgesia causes multiple adverse effects, delayed discharge, and unplanned readmissions. The use of regional techniques is therefore a rational approach to prevent and manage these problems.
\end{abstract}

This narrative review will focus on reviewing the available evidence (if any) to support the use of lower extremity peripheral regional anesthesia / analgesia techniques for surgeries commonly performed in the outpatient setting.

\title{
Cirugía Ambulatoria: ¿Dónde estamos?
}

La cirugía ambulatoria ya a estas alturas no es algo nuevo, y se ha consolidado como una forma costo-eficiente y segura de realizar múltiples tipos de cirugías.

Desde su origen, en la década de los '70, su crecimiento se consolidó en la década de los ochenta, particularmente en Estados Unidos (USA), en donde confluye el interés del sistema por la contención de costos asociados a la atención de Salud, en particular asociado a procedimientos quirúrgicos, con el desarrollo tecnológico que permite el advenimiento de la cirugía mínimamente invasiva. En 1982 Medicare comienza a cubrir procedimientos ambulatorios, acción que impulsa de forma importante la conversión de muchos procedimientos hospitalarios a ambulatorios. Durante la década de los '90 este crecimiento se acelera: en USA, el número de Centros de Cirugía Ambulatoria (ASC) pasó de poco menos de 1500 a casi 5000 en 2007. Actualmente más del 70\% de la cirugía ambulatoria en USA se realiza en una modalidad ambulatoria o de hospitalización abreviada.

Actualmente, ha habido un camino largo desde el primer ASC en Arizona, donde Ford y Reed instalaron su centro, realizando miringotomías pediátricas por 90 USD de la época . Las cirugías realizadas han ido ganando complejidad y se han ajustado a una lógica mínimamente invasiva.

Procedimientos que involucran estructuras como músculos, tendones y partes blandas (principalmente reparación artroscópica de manguito rotador o liberaciones de dedos en gatillo) son el segundo tipo más frecuente de cirugías ambulatorias realizadas en USA. Cirugías que involucran articulaciones (principalmente hombro y rodilla) son las terceras más frecuentes. Otros procedimientos frecuentemente realizados en un ambiente ambulatorio son cirugía de menisco de la rodilla, procedimientos óseos no asociados a fracturas o artroplastías, resecciones óseas parciales, o correcciones de deformidades de ortejos.

En ese contexto, una de las características más relevantes de las cirugías ambulatorias más frecuentes, es que pese a ser localizadas y frecuentemente realizadas usando tecnologías mínimamente invasivas, se asocian a un dolor postoperatorio significativo: en un estudio publicado el año 2013, que evaluaba la intensidad del dolor durante el primer día postoperatorio de más de 170 tipos distintos de cirugías realizados en 105 hospitales alemanes, encontraron que existen cirugías consideradas "poco invasivas", pero que se asocian a dolor significativo. En particular, de los 40 procedimientos más dolorosos, 22 eran ortopédicos, con numerosos procedimientos realizados en extremidades (superior e inferior) entre ellos. La artrodesis de tobillo ocupaba el séptimo lugar entre las cirugías más dolorosas, las reconstrucciones artroscópicas de ligamentos de la rodilla ocupaban el lugar 36, las correcciones quirúrgicas de metatarsos y ortejos el lugar 66, sólo por mencionar algunas.

Existen algunos conceptos básicos que rigen la filosofía de la cirugía ambulatoria: el primero es la seguridad de los pacientes, le siguen la eficiencia operacional y financiera. La razón de ser del éxito de la cirugía ambulatoria es la eficiencia con que compite con el sistema hospitalario. Uno de los pilares de esta eficiencia es la estandarización. Se busca utilizar fórmulas exitosas, técnicas altamente replicables, que permitan tener un resultado similar, con las mínimas desviaciones, y que aseguren un alto flujo de procedimientos. Esta eficiencia por una parte implica disminuir los costos directos asociados a hospitalizaciones, y por otro lado, aumentar el número de procedimientos realizables en horarios hábiles. Es por esto que el espectro de cirugías que se realizan en el contexto ambulatorio es bastante restringido.

Los principales enemigos de la cirugía ambulatoria son todas aquellas situaciones que retardan el alta, y/o favorecen las readmisiones no programadas. Las causas de esto son el manejo del dolor, las náuseas y vómitos postoperatorios.

En el escenario previamente descrito, nos encontramos entonces con cirugías que involucran las extremidades inferiores, principalmente ortopédicas, tanto óseas como de partes blandas, como dentro de las más frecuentemente real- 
izadas en el contexto ambulatorio. Estas cirugías, a su vez, están dentro de aquellas que se asocian potencialmente a dolor postoperatorio significativo durante las primeras 24 horas.

\section{Anestesia Regional para Cirugía Ambulatoria}

Todo lo anterior sienta condiciones muy propicias para considerar el uso de Anestesia Regional como uno de los elementos claves para el éxito de la cirugía ambulatoria: técnica segura, confiable, que permite una excelente calidad de analgesia, que disminuye el uso de drogas que potencialmente retardan el alta, por sedación excesiva, náuseas y vómitos postoperatorios, etc. .

Al comparar el uso de anestesia general con el uso de anestesia regional para cirugía ambulatoria, la evidencia demuestra que el uso de anestesia regional posee diversas potenciales ventajas: menor requerimiento de atención en la unidad de recuperación post-anestésica, menos incidencia de náuseas y menor dolor postoperatorio.

Sin embargo, al entrar en detalles respecto a las técnicas de anestesia regional disponibles, si bien las técnicas neuraxiales (anestesia espinal y/o peridural), presentan algunas características que las hacen menos indicadas en el contexto ambulatorio: por una parte, la elección de las drogas a utilizar, con la presencia de síntomas neurológicos transitorios (TNS), en particular con lidocaína espinal, y por otra, limitaciones propias de la técnica, como una regresión poco predecible del bloqueo, afectando la deambulación o la micción postoperatoria, el compromiso bilateral, y finalmente, la ausencia de una analgesia postoperatoria, lo que es un problema en el caso de procedimientos dolorosos.

\section{Anestesia Regional Periférica para Cirugía Ambulatoria}

Y acá estamos entonces: procedimientos ambulatorios cada vez más frecuentes, cuyo éxito requiere consistencia y alta eficiencia, que se concentran en extremidades y que requieren una analgesia postoperatoria que sea lo más confiable y selectiva posible. El uso de técnicas regionales periféricas entonces, se convierte en una consecuencia lógica de esta tendencia.

Nos abocaremos por tanto, a revisar aquellos procedimientos anestésicos y quirúrgicos que son más frecuentes en el contexto ambulatorio, enfocándonos en la evidencia que existe para su uso. En los casos en que corresponda, revisaremos las alternativas, cuáles son sus desventajas y desventajas.

A modo introductorio, podemos decir que una de las características de los bloqueos de extremidad inferior, que los diferencian de los de la extremidad superior, es que desde el punto de vista clínico, la anestesia regional periférica de la totalidad de la extremidad inferior requiere abordar al menos dos grandes plexos: el plexo lumbar y el plexo lumbosacro. Esto es posiblemente una de las razones por las que los bloqueos de extremidad inferior son menos utilizados por los anestesiólogos, dada la "competencia" que existe con las técnicas neuraxiales, que se entrenan más durante el período de la residencia.

Como veremos más adelante, esto tiene consecuencias prácticas, respecto a potenciales problemas asociados a los bloqueos de extremidad inferior. Dada la dificultad que representa la totalidad de una extremidad parcial o totalmente bloqueada para la deambulación, en los bloqueos de extremidad inferior, existe un balance que debe tenerse siempre en consideración, respecto al uso de bloqueos más proximales o extensos, que comprometen mayor cantidad de grupos musculares, afectando la estabilidad, con bloqueos más distales y selectivos, que pueden comprometer la calidad de la analgesia (Figura 1), haciendo más importante una aproximación multimodal.

Dado lo anterior, si bien todos los bloqueos de extremidad inferior descritos podrían ser utilizados para cirugía ambulatoria, se privilegian aquellos más selectivos, relegando algunos como los bloqueos de plexo lumbar, o ciáticos proximales, a algunos procedimientos específicos.

\section{Técnicas de neuro-localización para Anestesia Regional Periférica}

La evidencia disponible, basada en estudios de costo-efectividad, indican que el uso de ultrasonografía como técnica de neuro-localización aumenta la tasa de éxito de los procedimientos, su seguridad y en particular en el contexto ambulatorio, resulta desde el punto de vista del análisis económico de costo-eficiencia, más conveniente que el uso de la electro-estimulación.

\section{Cirugías y técnicas de bloqueos anestésicos/analgésicos de la Extremidad Inferior}


A continuación, nos referiremos a aspectos anestésicos de las cirugías de extremidad inferior más comúnmente realizadas en el contexto ambulatorio.

\section{Cirugía de Cadera}

Las cirugías artroscópicas de cadera son procedimientos ambulatorios realizados para el tratamiento de diversas patologías (pinzamiento, excisiones de bursas, liberación del psoas, por mencionar algunas). La intensidad del dolor postoperatorio puede ser significativa. Se han descrito diversos abordajes analgésicos usando bloqueos de nervio periférico. La cápsula articular de la cadera recibe inervación desde el nervio femoral, el obturador, el ciático, el nervio al cuadrado femoral y glúteo superior. Hay reportes usando bloqueos de plexo lumbar por abordaje posterior, bloqueos de cuadrado lumbar, bloqueos de fascia ilíaca, bloqueos femorales, y más recientemente, el bloqueo de PENG (Pericapsular Nerve Group), con diversos resultados.

Una revisión sistemática publicada el 2016 mostraba una notable falta de estudios controlados aleatorizados (RCT) en este tema, con reportes de casos, series clínicas y estudios comparativos no aleatorizados. Los reportes publicados evaluaban el efecto de los bloqueos femoral, fascia ilíaca, plexo lumbar por abordaje posterior y abordajes paravertebrales lumbares altos. Los estudios mostraban que el uso de bloqueos de nervio periférico se asociaba a una disminución significativa del dolor y el consumo de opioides cuando se agregaban al uso de anestesia general. También el 2016, Bech et al . realizó otra revisión sistemática evaluando opciones de manejo analgésico para artroscopías de cadera. La revisión incluyó $5 \mathrm{RCT}$, entre ellos estudios primarios que evaluaban infiltraciones peri-articulares con anestésicos locales (LIA) y bloqueos de nervio periférico. La calidad metodológica de los estudios era muy heterogénea, lo que finalmente impidió a los autores hacer recomendaciones claras respecto a una técnica analgésica en particular.

Un punto importante a considerar cuando se usan bloqueos de nervio periférico proximales, es el compromiso motor de la musculatura del muslo y el cuádriceps. Behrends et al. evaluó el efecto de usar bloqueos de fascia ilíaca en pacientes sometidos a artroscopías de cadera, a los cuales también se les administraba LIA. No hubo diferencias en términos de dolor en el postoperatorio inmediato entre ambos grupos, y el grupo que recibió el bloqueo de fascia ilíaca presentó disminución de la función motora del cuádriceps, lo que según los autores potencialmente puede aumentar el riesgo de caídas. Por otra parte, el procedimiento en sí tiene un riesgo no menor de presentar lesiones neurológicas. Dentro de los factores de riesgo asociados, se describen el tiempo y la intensidad de la tracción durante el procedimiento y el uso de bloqueos de fascia ilíaca, como un factor predictor.

En suma, la evidencia que avala el uso de bloqueos de nervio periférico en artroscopía de cadera no es categórica. Pese a mostrar beneficios en términos de menor dolor y consumo de opioides postoperatorios, el balance entre la magnitud del efecto, alcanzable con otras técnicas multimodales, en particular con LIA y el potencial riesgo de daño secundario a la realización de los bloqueos de nervio periférico, no permiten hacer recomendaciones en favor del uso de bloqueos en general, y de ninguna técnica en particular.

\section{Cirugía de Rodilla}

La cirugía artroscópica de rodilla y la reconstrucción de ligamentos de la rodilla son de los procedimientos más frecuentemente realizados en una modalidad ambulatoria. El dolor postoperatorio asociado a estos procedimientos es considerado moderado, con valores de escalas numéricas de dolor (NRS) en torno a 5.0 (0-10).3 El uso de anestesia y analgesia regional, en sus más diversas formas (neuraxial, bloqueos de distintos componentes del plexo lumbar), asociado a un abordaje analgésico multimodal, buscan optimizar la calidad de la analgesia, disminuir efectos colaterales, favorecer la deambulación y rehabilitación postoperatorias. Con el objeto de evaluar la efectividad de distintas técnicas analgésicas para cirugía de rodilla, y en particular para reconstrucción ligamentosa en el contexto ambulatorio, Secrist et al. el 2016 realizó una revisión sistemática, de 77 estudios aleatorizados (RCT). Los autores concluyeron que los bloqueos de nervio periférico serían igualmente efectivos que la inyección intra-articular de anestésicos locales como forma de analgesia. Esta revisión sistemática en particular analiza principalmente el bloqueo de nervio femoral (en inyección única o continuo) como abordaje al plexo lumbar. Cabe señalar que si bien es una revisión sistemática, no realizaron un meta-análisis que permitiera cuantificar la magnitud de los efectos observados, y por otra parte, no incluye estudios evaluando la efectividad de otras técnicas, como el bloqueo de canal aductor en cualquiera de sus variantes.

\section{Bloqueo Femoral (FNB)}


Una revisión sistemática publicada el 2019 evalúa precisamente la efectividad del bloqueo femoral como alternativa analgésica en cirugía ambulatoria de reconstrucción de ligamento cruzado anterior. La revisión se concentró en RCT comparando bloqueos femorales anestésicos o analgésicos, en inyección única o continuos, contra formas de analgesia multimodal, incluyendo la inyección intra-articular de anestésicos locales (LIA). Ocho estudios cumplieron criterios de inclusión en la Revisión Sistemática. Los autores concluyen que el uso de FNB tiene un efecto modesto en los resultados analgésicos, escalas de dolor durante las primeras 24 horas y tiempo para requerir el primer analgésico de rescate. Del mismo modo, al evaluar el uso de FNB en asociación a LIA, éste no mejoraba la calidad de la analgesia, comparado con LIA por sí sola. Es necesario hacer notar una serie de limitaciones del estudio. La más evidente es la falta de estudios comparables, y la heterogeneidad de ellos, que de hecho impidieron que se pudiese hacer un metaanálisis que entregara una cuantificación de los efectos indicados por los autores. De la información recopilada no se pudo concluir nada respecto al impacto del bloqueo de cuádriceps en los pacientes que recibieron un FNB.

Pese a que los autores indican que basados en sus resultados el FNB no agrega beneficios al esquema analgésico multimodal cuando éstos incluyen LIA, finalmente establecen que se necesitan más estudios primarios para confirmar esa conclusión.

\section{Bloqueo de Canal Aductor (ACB)}

Una revisión sistemática y meta-análisis publicada el 2019 evaluó el efecto analgésico del ACB en cirugía artroscópica de rodilla y en reconstrucciones artroscópicas de ligamento cruzado anterior (ACLR).

Los autores realizaron la revisión encontrando 10 RCT que cumplían con los criterios de inclusión. Los estudios comparaban ACB con bloqueo femoral o con placebo. Evaluaron como desenlaces relevantes la intensidad del dolor postoperatorio estático y dinámico, consumo de opioides, efectos adversos asociados a su uso, satisfacción usuaria, compromiso motor del cuádriceps, entre otras variables.

$\mathrm{Al}$ analizarse por separado los resultados de estudios que consideraban cirugías artroscópicas, el ACB disminuía el dolor postoperatorio durante las primeras 8 horas y el consumo de opioides durante las primeras 24 horas, pero en una magnitud muy modesta. $\mathrm{Al}$ analizarse el efecto del $\mathrm{ACB}$ en cirugía de reconstrucción de ligamento cruzado anterior, y compararse contra bloqueos femorales y placebo, el ACB no mejora los desenlaces analgésicos, contra ninguno de los dos comparadores.

Si bien los autores concluyen que el rol del ACB en la analgesia de la cirugía artroscópica de rodilla es limitada, en particular en la cirugía de reconstrucción de ligamento cruzado anterior, estos resultados deben interpretarse cuidadosamente, en especial considerando el número de estudios incluidos en el subgrupo ACLR, dado que la mayor parte del meta-análisis se realizó con un máximo de tres estudios, totalizando menos de 120 pacientes en las comparaciones con placebo y poco más de 300 pacientes en las comparaciones con FNB.

\section{IPACK Block (Interspace between the Popliteal Artery And posterior Capsule of the Knee)}

Este bloqueo ha captado la atención del mundo anestesiológico en las redes sociales. Tiene múltiples videos e información disponible en internet. Se describe como la infiltración de la zona posterior de la cápsula articular de la rodilla bajo guía ultrasonográfica. ,

La información disponible de estudios clínicos publicados tras revisión por pares es escasa. , Pareciera tener la ventaja de respetar la función motora, facilitando la rehabilitación. Su uso ha sido descrito en artroplastías de rodilla. Son necesarios estudios y series que reporten su uso en el contexto ambulatorio de cirugías de rodilla.

\section{Bloqueos combinados (Femoral y ciático)}

En el caso de cirugías complejas de rodilla realizadas en el contexto ambulatorio, como osteotomías, reconstrucciones multi-ligamentosas, reconstrucción de LCA, se ha descrito el uso de bloqueos combinados de nervio femoral y ciático. Cuando se compara contra bloqueos femorales, esta combinación tendría la ventaja de mejorar significativamente la analgesia, disminuir el consumo de opioides y la estadía en recuperación. Del mismo modo, también en cirugías complejas de rodilla, cuando se compara con bloqueos femorales (o no bloqueo), el uso combinado de bloqueos femoral y ciático se asocia además a menos readmisiones no programadas.

Sin embargo, en estos bloqueos combinados de nervios mixtos, el nivel de compromiso motor, sensitivo y propioceptivo es tan extenso y a veces tan intenso, que agrega una serie de dificultades al manejo ambulatorio de los pacientes: 
el cuidado de la extremidad insensible por períodos prolongados debe ser una consideración muy relevante por el riesgo de lesiones inadvertidas, y por sobre todo, el riesgo de caídas.

\section{Cirugía de Pierna y tobillo}

Procedimientos quirúrgicos distales a la rodilla se benefician del uso de bloqueos ciáticos, ya sea proximales o a nivel del hueco poplíteo. Cirugías como fracturas de tobillo o de huesos del pie (tarsos, metatarsos) pueden ser realizadas usando bloqueos combinados de nervio periférico como anestesia única, combinando un bloqueo ciático con un bloqueo femoral, ACB o safeno. En todas las técnicas (ciático subglúteo o ciático-poplíteo) se produce bloqueo sensitivo de toda la extremidad distal a la rodilla, con la excepción de la cara medial de la pierna y el maléolo medial, inervados por el nervio safeno (rama terminal del nervio femoral). El bloqueo ciático produce bloqueo motor de toda la musculatura distal a la rodilla, impidiendo la movilización del tobillo.

Los bloqueos ciáticos se han utilizado para anestesia/ analgesia en artroplastías y artroscopías de tobillo, reparación de ruptura de tendón de Aquiles, reducción abierta y fijación interna de fracturas de tibia y/o peroné, por mencionar las más frecuentemente realizadas en el contexto ambulatorio.

\section{Cirugía de Antepie (Hallux Valgus)}

La cirugía correctora de deformidades del antepie es una de las cirugías ambulatorias más frecuentes. Se asocia a dolor significativo durante las primeras 24-48 horas, por lo que se benefician de incluir técnicas de bloqueos regionales en el contexto de esquemas de analgesia multimodal. Bloqueos regionales a nivel ciático o de tobillo utilizando anestésicos de larga duración pueden proveer analgesia por 12-18 horas.

El 2020 se publicó la recomendación PROSPECT (PROcedure-SPECific postoperative pain managemenT) de la iniciativa PROSPECT para la cirugía de corrección de hallux valgus.

La iniciativa busca entregar recomendaciones basadas en evidencia específica, considerando riesgos y beneficios de las intervenciones, privilegiando estrategias multimodales y libres de opioides.

En el caso de la cirugía de hallux valgus, la recomendación considera el uso de analgesia multimodal asociada a bloqueos regionales. La revisión de la literatura no encontró estudios que evaluaran bloqueos ciático poplíteos que cumplieran los criterios necesarios para ser incluidos en la metodología PROSPECT. Los autores concluyen que los bloqueos de tobillo, en inyección única debiesen ser incluidos en el esquema de analgesia multimodal, junto a paracetamol, AINEs (i-COX-2) y corticoides sistémicos.

Una nota al margen respecto a esta recomendación es que pese a que los bloqueos ciático-poplíteos son comúnmente aprendidos como una alternativa para esta cirugía y frecuentemente realizados, 10,11 su uso carece de la evidencia metodológicamente apta para ser considerados en la guía. Este, como muchos otros ejemplos, es un llamado a llenar los vacíos de conocimiento que tenemos en nuestra práctica clínica habitual.

\section{Complicaciones asociadas al uso de Bloqueos de Extremidad Inferior en Cirugía Ambulatoria}

Como todo acto médico, los procedimientos regionales no están exentos de complicaciones. La gran mayoría son comunes al ámbito hospitalario: daño neurológico, toxicidad sistémica por anestésicos locales, sangramiento, sólo por mencionar algunos.

Sin embargo, hay algunas de ellas que son más propias en el contexto ambulatorio. Una de las más importantes es el riesgo de caídas.39-41

El tener una extremidad parcialmente insensible aumenta el riesgo de daño inadvertido, la alteración de propiocepción aumenta el riesgo de pérdidas de equilibrio y control, en especial cuando se agrega bloqueo motor, aunque sea parcial. Un estudio muy interesante, realizado por Muraskin et al. el 2007 evaluó el efecto de bloqueos femorales, ciáticos y la combinación de ambos en voluntarios en un laboratorio de análisis de movimiento. Los autores demostraron que el uso de bloqueos de nervio periférico de extremidad inferior disminuía significativamente la rigidez de la extremidad inferior y su estabilidad lateral, potencialmente afectando las maniobras de pivote. Esta observación en un estudio piloto enfatiza la importancia de una adecuada selección de los pacientes, el procedimiento y una buena educación de los pacientes y sus cuidadores, para prevenir este tipo de complicaciones. 
Poniendo las cosas en perspectiva: Tendencias en el uso de Anestesia Regional, en particular en el ámbito ambulatorio

Pese a lo anterior, la evidencia recogida muestra una gran dispersión respecto al uso de la anestesia regional. En USA, y pareciera que en otras partes también, pese a que existe una tendencia al aumento de su uso, aún la proporción de casos que se realizan con anestesia regional es muy baja, y de eso, una proporción aún menor es la que usa técnicas regionales en cirugía ambulatoria, en particular, para menos del 5\% de todos los casos en los que se pudiese haber usado un bloqueo de extremidad inferior.

En otras palabras, que estemos haciendo esta revisión de la literatura y presentando evidencia en favor del uso de la anestesia regional periférica para cirugía ambulatoria de extremidad inferior, debiese servir para estimular su uso, y aumentar la proporción de procedimientos que la incorporan.

\section{Conclusiones}

El uso de bloqueos de extremidad inferior puede proveer de muchos beneficios para los pacientes sometidos a cirugía ambulatoria. Le elección racional de las técnicas a emplear, basadas en la evidencia disponible es fundamental para obtener buenos resultados. El uso de tecnologías como la ultrasonografía han aumentado significativamente la eficiencia de los procedimientos regionales en el contexto ambulatorio. Sin embargo, existe una necesidad por mejorar nuestro conocimiento respecto a cuáles son las técnicas más indicadas, basados en la realización de estudios primarios metodológicamente aptos y sintetizando la información de una manera no sesgada.
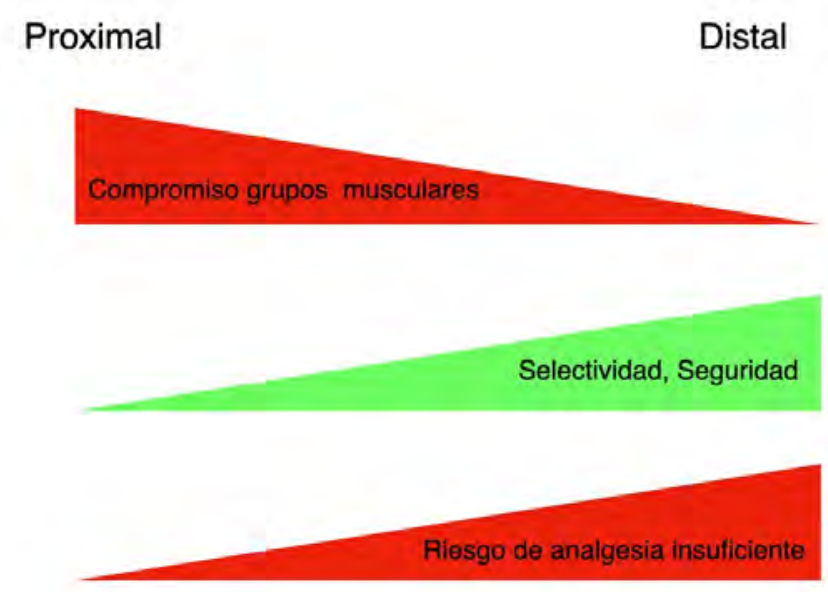

Figura 1. Balance entre la extensión anatómica de los bloqueos de nervio periférico, su efecto sobre grupos musculares, selectividad y riesgo de analgesia insuficiente.

\section{Referencias}

1 Carey, K., Burgess, J., Young, G. (2011). Hospital competition and financial performance: the effects of ambulatory surgery centers. Health Economics 20(5), 571-581.

2 Steiner CA, Karaca Z, Moore BJ, Imshaug MC, Pickens G. Surgeries in Hospital-Based Ambulatory Surgery and Hospital Inpatient Settings, 2014. HCUP Statistical Brief \#223. May 2017. Agency for Healthcare Research and Quality, Rockville, MD. 
3 Gerbershagen, H., Aduckathil, S., Wijck, A., Peelen, L., Kalkman, C., Meissner, W. (2013). Pain intensity on the first day after surgery: a prospective cohort study comparing 179 surgical procedures. Anesthesiology 118(4), 934-44.

4 Gold, B. (1989). Unanticipated admission to the hospital following ambulatory surgery JAMA: The Journal of the American Medical Association 262(21), 3008-3010.

5 Kopp, S., Horlocker, T. (2010). Regional anaesthesia in day-stay and short-stay surgery Anaesthesia 65(s1), 84-96.

6 Liu, S., Strodtbeck, W., Richman, J., Wu, C. (2005). A Comparison of Regional Versus General Anesthesia for Ambulatory Anesthesia: A Meta-Analysis of Randomized Controlled Trials Anesthesia \& Analgesia 101(6), 1634-1642.

7 Schneider, M., Ettlin, T., Kaufmann, M., Schumacher, P., Urwyler, A., Hampl, K., Hochstetter, A. (1993). Transient Neurologic Toxicity After Hyperbaric Subarachnoid Anesthesia with 5\% Lidocaine. Anesthesia \& Analgesia 76(5), 1154.

8 Mulroy, M., McDonald, S. (2003). Regional anesthesia for outpatient surgery Anesthesiology Clinics of North America 21(2), 289-303.

9 Hadžić, A., Vloka, J. D., Kuroda, M. M., Koorn, R., \& Birnbach, D. J. (1998). The practice of peripheral nerve blocks in the United States: A national survey. Regional anesthesia and pain medicine, 23(3), 241-246.

10 Corvetto, M., Echevarria, G., Espinoza, A., Altermatt, F. (2015). Which types of peripheral nerve blocks should be included in residency training programs? BMC Anesthesiology 15(1), 32.4

11 Corvetto, M., Carmona, J., Vásquez, M., Salgueiro, C., Crostón, J., Sosa, R., Folle, V., Altermatt, F. (2017). Current practice in regional anaesthesia in South America: An online survey Revista Española de Anestesiología y Reanimación (English Edition) 64(1), 27-31.

12 Chou, R., Gordon, D., Leon-Casasola, O., Rosenberg, J., Bickler, S., Brennan, T., Carter, T., Cassidy, C., Chittenden, E., Degenhardt, E., Griffith, S., Manworren, R., McCarberg, B., Montgomery, R., Murphy, J., Perkal, M., Suresh, S., Sluka, K., Strassels, S., Thirlby, R., Viscusi, E., Walco, G., Warner, L., Weisman, S., Wu, C. (2016). Management of Postoperative Pain: A Clinical Practice Guideline From the American Pain Society, the American Society of Regional Anesthesia and Pain Medicine, and the American Society of Anesthesiologists' Committee on Regional Anesthesia, Executive Committee, and Administrative Council The Journal of Pain 17(2), 131-157.

13 Ehlers, L., Jensen, J., Bendtsen, T. (2012). Cost-effectiveness of ultrasound vs nerve stimulation guidance for continuous sciatic nerve block BJA: British Journal of Anaesthesia 109(5), 804-808.

14 Phillips, D., Knizner, T., Williams, B. (2011). Economics and Practice Management Issues Associated With Acute Pain Management Anesthesiology Clinics 29(2), 213-232.

15 Liu, S., John, R. (2010). Modeling Cost of Ultrasound Versus Nerve Stimulator Guidance for Nerve Blocks With Sensitivity Analysis Regional Anesthesia and Pain Medicine 35(1), 57-63.

16 Baker, J. F., Byrne, D. P., Hunter, K., \& Mulhall, K. J. (2011). Post-operative opiate requirements after hip arthroscopy. Knee surgery, sports traumatology, arthroscopy : official journal of the ESSKA, 19(8), $1399-1402$.

17 Birnbaum, K., Prescher, A., Heßler, S., Heller, K. (1997). The sensory innervation of the hip joint - an anatomical study Surgical and Radiologic Anatomy 19(6), 371-375.

18 YaDeau, J. T., Tedore, T., Goytizolo, E. A., Kim, D. H., Green, D. S., Westrick, A., Fan, R., Rade, M. C., Ranawat, A. S., Coleman, S. H., \& Kelly, B. T. (2012). Lumbar plexus blockade reduces pain after hip arthroscopy: a prospective randomized controlled trial. Anesthesia and analgesia, 115(4), 968-972.

19 Kinjo S, Kolodzie K, Dong K, Zhang AL. The effects of transmuscular quadratus lumborum blocks on postoperative pain in arthroscopic hip surgery: a cohort analysis. J Anesth. 2019;33(4):516-522.

20 Badiola, I., Liu, J., Huang, S., Kelly, J. D., 4th, \& Elkassabany, N. (2018). A comparison of the fascia iliaca block to the lumbar plexus block in providing analgesia following arthroscopic hip surgery: A randomized controlled clinical trial. Journal of clinical anesthesia, 49, 26-29.

21 Ward, J., Albert, D., Altman, R., Goldstein, R., Cuff, G., Youm, T. (2012). Are Femoral Nerve Blocks Effective for Early Postoperative Pain Management After Hip Arthroscopy? Arthroscopy: The Journal of Arthroscopic \& 
Related Surgery 28(8), 1064-1069.

22 Orozco, S., Muñoz, D., Jaramillo, S., Herrera, A. (2020). Pericapsular Nerve Group (PENG) block for perioperative pain control in hip arthroscopy Journal of Clinical Anesthesia 59(), 3-4.

23 Kay, J., SA, D., Memon, M., Simunovic, N., Paul, J., Ayeni, O. (2016). Examining the Role of Perioperative Nerve Blocks in Hip Arthroscopy: A Systematic Review Arthroscopy: The Journal of Arthroscopic \& Related Surgery 32(4), 704-715.e1.

24 Bech, N., Hulst, A., Spuijbroek, J., Leuken, L., Haverkamp, D. (2016). Perioperative pain management in hip arthroscopy; what options are there? Journal of Hip Preservation Surgery 3(3), 181-189.

25 Behrends, M., Yap, E., Zhang, A., Kolodzie, K., Kinjo, S., Harbell, M., Aleshi, P. (2018). Preoperative Fascia Iliaca Block Does Not Improve Analgesia after Arthroscopic Hip Surgery, but Causes Quadriceps Muscles Weakness Anesthesiology 129(3), 536-543.

26 Bailey, T., Stephens, A., Adeyemi, T., Xu, Y., Presson, A., Aoki, S., Maak, T. (2019). Traction Time, Force and Postoperative Nerve Block Significantly Influence the Development and Duration of Neuropathy Following Hip Arthroscopy Arthroscopy: The Journal of Arthroscopic \& Related Surgery 35(10), 2825-2831.

27 Moore, J., Ross, S., Williams, B. (2013). Regional anesthesia and ambulatory surgery Current Opinion in Anaesthesiology 26(6), 652-660.

28 Memtsoudis, S. G., Kuo, C., Ma, Y., Edwards, A., Mazumdar, M., \& Liguori, G. (2011). Changes in anesthesiarelated factors in ambulatory knee and shoulder surgery: United States 1996-2006. Regional anesthesia and pain medicine, 36(4), 327-331.

29 McCartney, C., McIsaac, D. (2019). Are Peripheral Nerve Blocks Indicated in Ambulatory Knee Surgery? Anesthesia \& Analgesia 128(1), 3-4.

30 Secrist, E. S., Freedman, K. B., Ciccotti, M. G., Mazur, D. W., \& Hammoud, S. (2016). Pain Management After Outpatient Anterior Cruciate Ligament Reconstruction: A Systematic Review of Randomized Controlled Trials. The American journal of sports medicine, 44(9), 2435-2447.

31 Vorobeichik, L., Brull, R., Joshi, G., Abdallah, F. (2019). Evidence Basis for Regional Anesthesia in Ambulatory Anterior Cruciate Ligament Reconstruction Anesthesia \& Analgesia 128(1), 58-65.

32 Sehmbi, H., Brull, R., Shah, U., El-Boghdadly, K., Nguyen, D., Joshi, G., Abdallah, F. (2019). Evidence Basis for Regional Anesthesia in Ambulatory Arthroscopic Knee Surgery and Anterior Cruciate Ligament Reconstruction Anesthesia \& Analgesia 128(2), 223-238.

33 Sinha SK. How I do it: Infiltration between Popliteal Artery and Capsule of the Knee (iPACK). ASRA newsletter; February 2019. https://www.asra.com/asra-news/article/158/how-i-do-it-infiltration-between-poplite

34 Sinha, S., Clement, A., Surette, A. (2019). Infiltration Between the Popliteal Artery and Capsule of the Knee (iPACK): Essential Anatomy, Technique, and Literature Review Current Anesthesiology Reports 9(4), 474-478.

35 Sankineani, S., Reddy, A., Eachempati, K., Jangale, A., Reddy, A. (2018). Comparison of adductor canal block and IPACK block (interspace between the popliteal artery and the capsule of the posterior knee) with adductor canal block alone after total knee arthroplasty: a prospective control trial on pain and knee function in immediate postoperative period European Journal of Orthopaedic Surgery \& Traumatology 28(7), 1391-1395.

36 Kim, D., Beathe, J., Lin, Y., YaDeau, J., Maalouf, D., Goytizolo, E., Garnett, C., Ranawat, A., Su, E., Mayman, D., Memtsoudis, S. (2018). Addition of Infiltration Between the Popliteal Artery and the Capsule of the Posterior Knee and Adductor Canal Block to Periarticular Injection Enhances Postoperative Pain Control in Total Knee Arthroplasty Anesthesia \& Analgesia 2019;129:526-35.

37 Harbell, M., Cohen, J., Kolodzie, K., Behrends, M., Braehler, M., Kinjo, S., Feeley, B., Aleshi, P. (2016). Combined preoperative femoral and sciatic nerve blockade improves analgesia after anterior cruciate ligament reconstruction: a randomized controlled clinical trial Journal of Clinical Anesthesia 33(), 68-74.

38 Williams, B., Kentor, M., Vogt, M., Williams, J., Chelly, J., Valalik, S., Harner, C., Fu, F. (2003). FemoralSciatic Nerve Blocks for Complex Outpatient Knee Surgery Are Associated with Less Postoperative Pain Before Same-day Discharge Anesthesiology 98(5), 1206-1213.

39 Williams, B., Kentor, M., Bottegal, M. (2007). The Incidence of Falls at Home in Patients with Perineural Femoral Catheters\&colon; A Retrospective Summary of a Randomized Clinical Trial Anesthesia \& Analgesia 
104(4), 1002.

40 Ilfeld, B. M., Duke, K. B., \& Donohue, M. C. (2010). The association between lower extremity continuous peripheral nerve blocks and patient falls after knee and hip arthroplasty. Anesthesia and analgesia, 111(6), 15521554.

41 Finn, D. M., Agarwal, R. R., Ilfeld, B. M., Madison, S. J., Ball, S. T., Ferguson, E. J., Morgan, A. C., \& Morris, B. A. (2016). Fall Risk Associated with Continuous Peripheral Nerve Blocks Following Knee and Hip Arthroplasty. Medsurg nursing : official journal of the Academy of Medical-Surgical Nurses, 25(1), 25-49.

42 Klein, S. M., Evans, H., Nielsen, K. C., Tucker, M. S., Warner, D. S., \& Steele, S. M. (2005). Peripheral nerve block techniques for ambulatory surgery. Anesthesia and analgesia, 101(6), 1663-1676.

43 Ardon, A., Prasad, A., McClain, R., Melton, M., Nielsen, K., Greengrass, R. (2019). Regional Anesthesia for Ambulatory Anesthesiologists Anesthesiology Clinics 37(2), 265-287.

44 Mulroy, M., McDonald, S. (2003). Regional anesthesia for outpatient surgery Anesthesiology Clinics of North America 21(2), 289-303.

45 https://esraeurope.org/about-prospect-copy/

46 Korwin-Kochanowska, K., Potié, A., El-Boghdadly, K., Rawal, N., Joshi, G., Albrecht, E., Collaboration, P. (2020). PROSPECT guideline for hallux valgus repair surgery: a systematic review and procedurespecific postoperative pain management recommendations Regional Anesthesia \& Pain Medicine https:// dx.doi.org/10.1136/rapm-2020-101479

47 Muraskin, S., Conrad, B., Zheng, N., Morey, T., Enneking, F. (2007). Falls Associated with Lower-ExtremityNerve Blocks: A Pilot Investigation of Mechanisms Regional Anesthesia and Pain Medicine 32(1), 67-72.

48 Joshi, G., Kehlet, H., Rawal, N. (2019). Surgeon-administered regional analgesia to replace anaesthetistadministered regional analgesia: need for communication and collaboration British Journal of Anaesthesia 123(6), 707-709.

49 Cozowicz, C., Poeran, J., Zubizarreta, N., Mazumdar, M., Memtsoudis, S. (2016). Trends in the Use of Regional Anesthesia Regional Anesthesia and Pain Medicine 41(1), 43-49.

50 Gabriel, R., Ilfeld, B. (2018). Use of Regional Anesthesia for Outpatient Surgery Within the United States Anesthesia \& Analgesia 126(6), 2078-2084. 